\title{
Convenient synthetic method of starch/lactic acid graft copolymer catalyzed with sodium hydroxide
}

\author{
QINGLING WANG, YINGMO HU*, JIANHUA ZHU, YANG LIU, XUE YANG and JING BIAN \\ School of Material Science and Engineering, China University of Geosciences, Beijing 100083, China
}

MS received 8 March 2011; revised 9 July 2011

\begin{abstract}
Copolymer of starch grafted with lactic acid (LA) could be directly prepared by reaction of cornstarch with lactic acid and with sodium hydroxide $(\mathrm{NaOH})$ as the catalyst. The structure of starch/LA copolymer was characterized by IR, XRD, SEM and ${ }^{1} \mathrm{H}-\mathrm{NMR}$. The effects of $\mathrm{NaOH}$ concentration, ratios of starch and LA, reaction temperature and reaction time on the grafting degree were also investigated and the results showed that the highest grafting degree of starch could reach $33.60 \%$ when the graft copolymerization was carried out in $0.40 \mathrm{~mol}^{-1} \mathrm{NaOH}^{-1}$ aqueous solution for $9 \mathrm{~h}$ at $90^{\circ} \mathrm{C}$ with 1: 6 ratio of starch and lactic acid.
\end{abstract}

Keywords. Starch; lactic acid; graft copolymerization.

\section{Introduction}

With the rapid development of plastic industry, 'white pollution' has become more and more serious, so more attention has been paid to the research and development of biodegradable polymers of sustainable materials for environmental protection. In many biodegradable polymers, starch is a potentially useful and completely biodegradable material for biodegradable plastics because of its nontoxic, low cost and its natural abundance which can be obtained from many crops including corn, wheat, rice, potato and so on (Tester and Karkallas 2002). Therefore, modification of starch, physically and chemically, has been extensively studied for the environmental and ecological concerns. Chemical modification, which can produce various starch-based products including chemical derivation and graft copolymerization, is the most widely used, and graft copolymerization, as a rational approach of chemical modification, has been extensively used in the modification of starch (Park and Inagaki 2003; Rouilly et al 2004; Gong et al 2006a). At present, grafted monomers are the main types of vinyl monomers and aliphatic polyester, although the copolymers of starch are grafted by vinyl monomers (Athawale and Lele 2000; Cho and Lee 2002; Zhai et al 2002; Fanta et al 2004) can improve the mechanical properties of starch and they do not biodegrade completely. However, starch graft copolymers by aliphatic polyester (Sang et al 1997; Choi et al 1999; Dubois et al 1999) are a kind of completely biodegradable polymer materials, but the coverage on them is limited. Recently, the copolymer of starch with lactic acid have been studied widely for its excellent degradability and the starch/LA copolymer has been prepared using different methods such

\footnotetext{
*Author for correspondence (huyingmo@ cugb.edu.cn)
}

as ring-opening polymerization of lactide in the presence of stannous 2-ethyl-hexanoate (Gong et al 2006b), ring-opening polymerization of lactide initiated by potassium tert-butoxide (Shao and Zhao 2009), introducing some protective groups and substituent groups to the raw materials and then further reaction (Chen et al 2005; Shi et al 2005; Xie et al 2006) and so on, However, either the procedures are very complex or the grafting degree is too low, so study on the starch/LA copolymer needs to be further investigated.

In this study, a convenient method was introduced to synthesize starch/LA copolymer by the reaction of starch with lactic acid directly catalyzed with $\mathrm{NaOH}$, the structure of starch/lactic acid copolymer was affirmed by means of IR, XRD, SEM and ${ }^{1} \mathrm{H}-\mathrm{NMR}$.

\section{Experimental}

$5 \mathrm{~g}$ of raw cornstarch was dispersed in $30 \mathrm{ml} 0.40 \mathrm{~mol} \mathrm{l}^{-1}$ $\mathrm{NaOH}$ (AR) aqueous solution and the mixture was heated to $75^{\circ} \mathrm{C}$ for gelatinization for about $1 \mathrm{~h}$, then $15 \mathrm{ml}$ dimethyl sulfoxide (AR) and $25 \mathrm{ml}$ LA (AR) were added to the flask. Graft copolymerization was carried out at $90^{\circ} \mathrm{C}$ under about $0.095 \mathrm{MPa}$ for $9 \mathrm{~h}$. Then the system was cooled to room temperature, and the product was washed twice with acetone (AR) under vigorous stirring to remove unreacted LA monomer, and dried in a vacuum oven at $80^{\circ} \mathrm{C}$ for $12 \mathrm{~h}$. And then the dried product was further purified by Soxhlet extraction with acetone for $48 \mathrm{~h}$ to remove the unreacted LA monomer completely as well as the PLA homopolymer. The final product was dried at $80^{\circ} \mathrm{C}$ under vacuum oven with a grafting degree of $33.60 \%$. The grafting degree is calculated as follows:

$$
\text { Grafting degree }=\frac{m_{1}-m_{0}}{m_{0}},
$$


where $m_{0}$ is the weight of dried raw starch and $m_{1}$ the weight of purified starch/LA copolymer.

The sample and cornstarch were characterized by IR (Perkin-Elmer 100) with the wave range from 4000 to $400 \mathrm{~cm}^{-1}$, XRD (D/max-Ra diffractometer) with $\mathrm{CuK}_{\alpha}$ radiation $(\lambda=1.5406 \AA)$ operating at $40 \mathrm{kV}$ and $100 \mathrm{~mA}$ and the sample was scanned from $3-50^{\circ}$ of $2 \theta$ by steps of $0.02^{\circ}$, and ${ }^{1} \mathrm{H}-\mathrm{NMR}$ (Bruker AV-400) with DMSO- $d_{6}$ as solvents. The morphology of sample and cornstarch was investigated by SEM (LEO-1450).

\section{Results and discussion}

From the IR spectra of starch (figure 1A), the strong and broad absorption peak at $3430 \mathrm{~cm}^{-1}$ is the characteristic absorption peak of the stretching vibration of $-\mathrm{OH}$, the peaks at $2935 \mathrm{~cm}^{-1}, 1644 \mathrm{~cm}^{-1}$ and $1160-1010 \mathrm{~cm}^{-1}$ are respectively assigned to the vibrational absorption of $\mathrm{C}-\mathrm{H}$ bond, intramolecular hydrogen bond and $\mathrm{C}-\mathrm{O}$ bond in starch, while the peaks at $570.6 \mathrm{~cm}^{-1}, 762.5 \mathrm{~cm}^{-1}$ and $862.4 \mathrm{~cm}^{-1}$ are the swing vibration absorption peaks of $\mathrm{C}-\mathrm{H}$ bond of starch. Compared with the spectrum of starch (figure 1A), the new strong absorption peaks at $1751 \mathrm{~cm}^{-1}$ and $2990 \mathrm{~cm}^{-1}$ are assigned to carbonyl of ester and $\mathrm{C}-\mathrm{H}$ bond in poly(lactic acid) chain, appearing in the IR spectrum of starch/lactic acid copolymer (figure 1B), and the other peaks almost did not change, which indicates that the starch has been successfully grafted by the lactic acid, and starch/LA copolymer has been formed.

The X-ray diffraction pattern of starch (figure 2A) shows diffraction peaks at $2 \theta=15 \cdot 8^{\circ}, 19 \cdot 3^{\circ}$ and $21.6^{\circ}$ which demonstrated good crystallizability of starch. Compared with the acute diffraction peaks of the starch, the starch/lactic acid copolymer (figure 2B) shows a broad dispersion peak at $2 \theta=18.7^{\circ}$, which indicates the starch/lactic acid copolymer is a kind of amorphous polymer, and the aggregation of starch has been changed and the original diffraction peaks completely disappeared after graft copolymerization of starch with lactic acid.

The scanning electron microscopic images of starch (A) and starch/lactic acid copolymer (B) are shown in

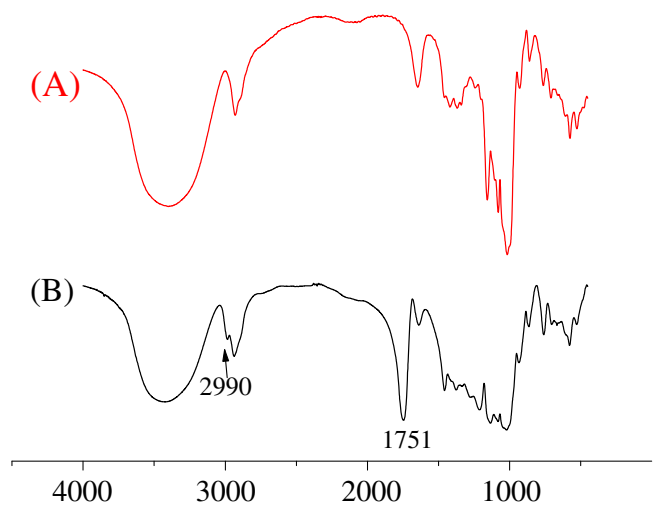

Figure 1. IR spectra of starch (A) and starch/LA copolymer (B).

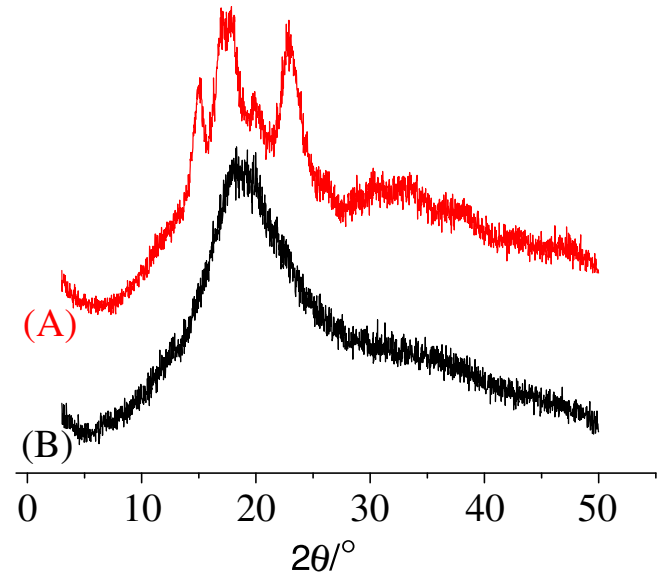

Figure 2. X-ray diffraction patterns of starch (A) and starch/LA copolymer (B).

figure 3. The particles of starch are spherical solid and regular, and have smooth surfaces and edges (figure $3 \mathrm{~A}$ ). Its surface has remarkable changes after modification becoming loose and irregular, because the external surfaces of starch have suffered certain corrosion from LA.

${ }^{1} \mathrm{H}-\mathrm{NMR}$ spectra of cornstarch and starch/LA copolymer are shown in figure 4. Seen from cornstarch (figure 4A), the signals at 5.40, 5.50 and $4.59 \mathrm{ppm}$ are respectively assigned to proton signals of hydroxyl group on $\mathrm{C}-2, \mathrm{C}-3$ and $\mathrm{C}-6$, while the proton signals assigned to $\mathrm{CH}$ and $\mathrm{CH}_{2}$ of the starch unit are centred at about 3.60$3.40 \mathrm{ppm}$. Seen from the ${ }^{1} \mathrm{H}-\mathrm{NMR}$ spectra of starch/LA copolymer (figure 4B), the signals at 5.11 and $4.18 \mathrm{ppm}$ are respectively assigned to proton signals of terminal hydroxyl group $\left(H_{f}\right)$ and the carbon adjacent to the terminal hydroxyl group $\left(H_{e}\right)$, while the proton signals assigned to the starch unit and $\mathrm{CH}$ of poly(lactic acid) chain $\left(H_{d}\right)$ are centred at $3.65-3.38 \mathrm{ppm}$, and the proton signals corresponding to $H_{c}$, $H_{b}$ and $H_{a}$ on the methyl groups of poly(lactic acid) chain are set at about $1.45,1.27$ and $1.08 \mathrm{ppm}$. So the ${ }^{1} \mathrm{H}-\mathrm{NMR}$ analysis could demonstrate the microstructure of starch/LA copolymer.

The mechanism of grafting polymerization reaction of starch with LA is inferred and shown in figure 5. By mixing the dried starch with $\mathrm{NaOH}$ aqueous solution so that the starch can be fully gelatinized by water in $\mathrm{NaOH}$ aqueous solution and the hydroxyl groups on the starch can also be activated by $\mathrm{NaOH}$ to form ion-pair, which produce the reactive sites of polymerization reaction. Our previous study has shown that LA can be transformed into lactide under mild conditions (Gong et al 2006a, b). The ring of lactide can be opened and its terminal group formed ion-pair of sodium alkoxide via ester exchange reaction of oxygen anion on the ion-pair generated by the starch with carbonyl group of lactide. The ion-pair of sodium alkoxide continues to react with the carbonyl group of lactide and the polyester chain is prolonged, producing the long chain of polylactic acid on the bone of starch. 

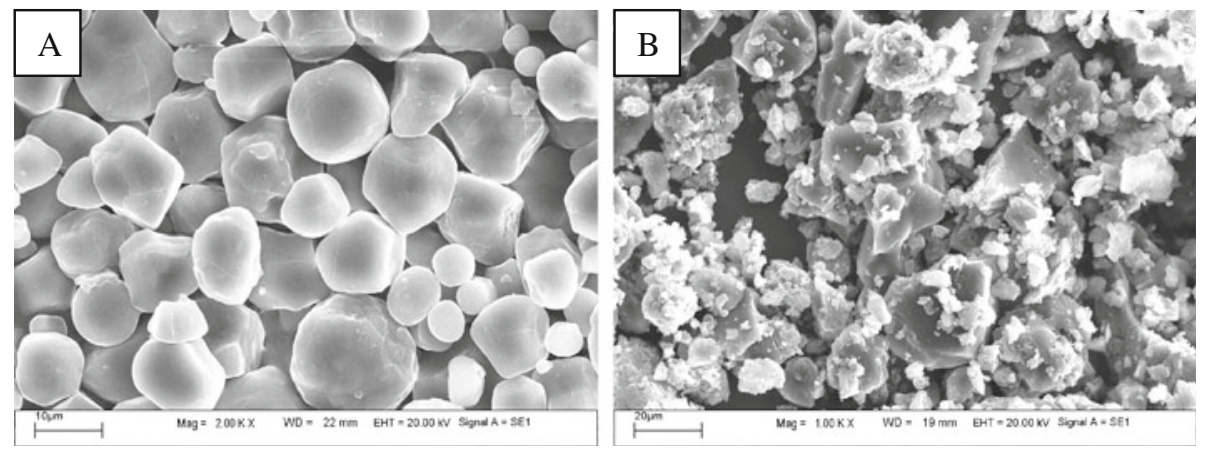

Figure 3. SEM images of starch (A) and starch/LA copolymer (B).

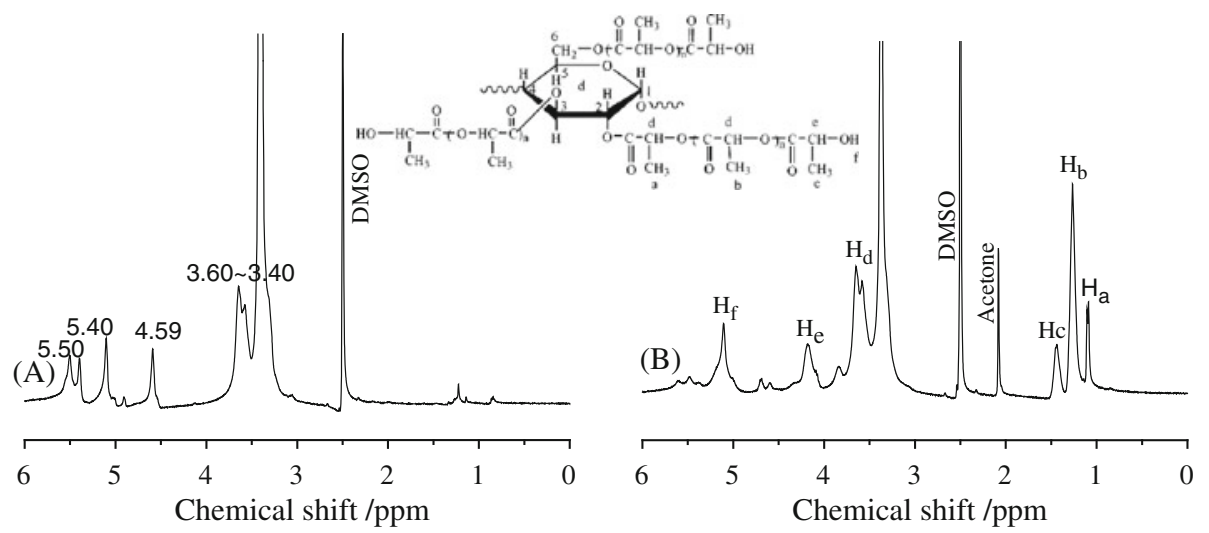

Figure 4. ${ }^{1} \mathrm{H}-\mathrm{NMR}$ spectra of cornstarch $(\mathbf{A})$ and $\mathrm{starch} / \mathrm{LA}$ copolymer $(\mathbf{B})$.

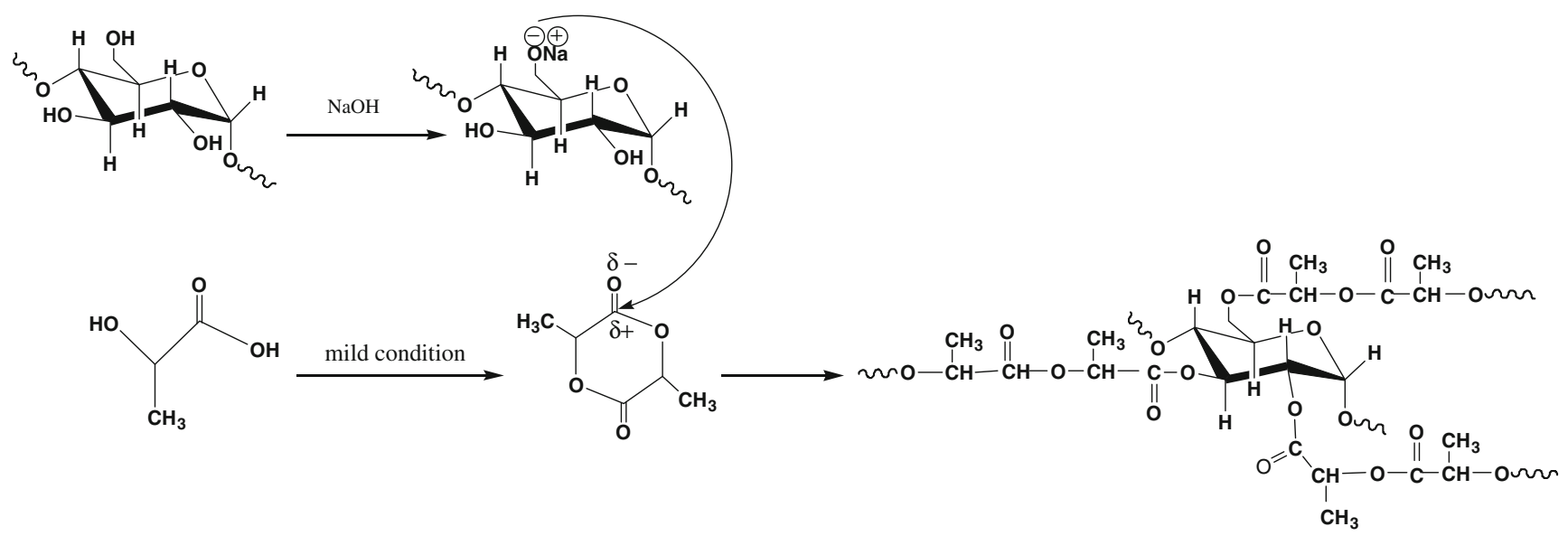

Figure 5. Reaction process of starch graft with lactic acid. 


\section{Conclusions}

In conclusion, starch/LA copolymer can be successfully synthesized by direct reaction of starch with lactic acid catalyzed with $\mathrm{NaOH}$ aqueous solution. Its structure is also confirmed by IR, XRD, SEM and ${ }^{1} \mathrm{H}-\mathrm{NMR}$, and the grafting degree of starch could reach $33.6 \%$ in experimental conditions.

\section{Acknowledgement}

This work is supported by the Fundamental Research Funds for the Central Universities (2011PY0171).

\section{References}

Athawale V D and Lele V 2000 Carbohydr. Polym. 41407 Chen L, Qiu X Y and Deng M X 2005 Polymer 465723
Cho C G and Lee K 2002 Carbohydr. Polym. 48125

Choi E J, Kim C H and Park J K 1999 Macromolecules 327402

Dubois P, Krishnan M and Narayan R 1999 Polymer 403091

Fanta G F, Felker F C and Shogren R L 2004 Carbohydr. Polym. 5677

Gong Q X, Tu K H and Wang L Q 2006a Acta Polym. Sin. 91045

Gong Q X, Wang L Q and Tu K H 2006b Carbohydr. Polym. 64 501

Park Y W and Inagaki N 2003 Polymer 441569

Rouilly A, Rigal L and Gilbert R G 2004 Polymer 457813

Sang H L, Kwang H L and Sung K H 1997 J. Appl. Polym. Sci. 64 1999

Shao J and Zhao Y M 2009 China Plastics 2315

Shi H J, Li A D and Liu Z Z 2005 J. Shenyang Inst. Chem. Technol. 19200

Tester R F and Karkallas J 2002 Starch 53513

Xie D M, Shi Y F and Xie C L 2006 J. Mater. Sci. Eng. 24836

Zhai M, Yoshii F and Kume T 2002 Carbohydr. Polym. 50295 